\title{
Serum concentration of eicosapentaenoic acid is associated with cognitive function in patients with coronary artery disease
}

Shusuke Yagi ${ }^{i^{*}+}$, Tomoya Hara ${ }^{1 \dagger}$, Rie Ueno ${ }^{1}$, Ken-ichi Aihara ${ }^{2}$, Daiju Fukuda ${ }^{3}$, Akira Takashima ${ }^{1}$, Junko Hotchi ${ }^{1}$, Takayuki Ise ${ }^{1}$, Koji Yamaguchi', Takeshi Tobiume ${ }^{1}$, Takashi Iwase ${ }^{1}$, Hirotsugu Yamada', Takeshi Soeki', Tetsuzo Wakatsuki ${ }^{1}$, Michio Shimabukuro ${ }^{3}$, Masashi Akaike $^{4}$ and Masataka Sata ${ }^{1}$

\begin{abstract}
Background: Recent studies have shown that intake of $n-3$ polyunsaturated fatty acids (PUFAs) is associated with reduced risk of cognitive impairment and coronary artery disease (CAD); however, it is currently unknown whether reduced serum n-3 PUFA is associated with cognitive impairment in patients with CAD.

Methods: We retrospectively evaluated cognitive function with the mini-mental state examination (MMSE), serum levels of PUFAs (including eicosapentaenoic acid [EPA], docosahexaenoic acid [DHA], dihomogammalinolenic acid [DGLA], and arachidonic acid [AA]), cardiovascular risk factors (hypertension, dyslipidemia, diabetes mellitus, cerebrovascular disease, and history of current/previous smoking), and parameters of cardiac function (left ventricular ejection fraction and brain natriuretic peptide levels) in 146 Japanese CAD patients. The associations between the MMSE scores and the other parameters were evaluated.
\end{abstract}

Results: Pearson correlation analysis showed that EPA $(R=0.25, P<0.01)$, EPA/AA ratio $(R=0.22, P=0.01)$, and left ventricular ejection fraction $(R=0.15, P=0.04)$ were positively associated with MMSE score, and that age $(R=-0.20$, $P<0.01)$ and brain natriuretic peptide levels $(R=-0.28, P<0.01)$ were inversely associated with MMSE score. Multiple regression analysis showed that age $(P<0.05)$ was negatively associated with MMSE score, while EPA $(P<0.01)$ and EPA/AA ratio $(P<0.05)$ were positively associated with MMSE score; however, sex; body mass index; left ventricular ejection fraction; levels of DHA, AA, and DGLA; DHA/AA ratio; brain natriuretic peptide; and presence of hypertension, dyslipidemia, diabetes mellitus, cerebrovascular disease, and history of current/previous smoking were statistically excluded.

Conclusions: Serum EPA concentration is associated with cognitive function in patients with CAD, suggesting that a low serum EPA level is a risk factor for cognitive impairment independent of cardiac function, including left ventricular ejection fraction. This correlation potentially lends further support to a role of dietary n-3 PUFAs in preventing the cognitive decline in CAD patients.

Keywords: Eicosapentaenoic acid, n-3 polyunsaturated fatty acids, Cognitive function, Mini-mental state examinations, Coronary artery disease

\footnotetext{
*Correspondence: syagi@tokushima-u.ac.jp

${ }^{\dagger}$ Equal contributors

'Department of Cardiovascular Medicine, The University of Tokushima Graduate School of Health Biosciences, 3-18-15 Kuramoto-cho, Tokushima-city, Tokushima 770-8503, Japan

Full list of author information is available at the end of the article
} 


\section{Background}

Cardiovascular disease has recently been implicated as a major factor in the development of dementia, as these diseases may be linked by shared common risks and pathogenic elements [1,2]. Accumulation of cardiovascular risk factors therefore leads to cognitive impairment. In addition, hypoxia/ischemia resulting from reduced cerebral blood flow due to cardiac dysfunction may be associated with dementia. Conversely, dementia itself could be an independent cardiovascular risk factor; patients with dementia have lifestyle-related problems, such as inappropriate food or alcohol intake, sedentary activity, and psychosocial stress, including depression [3]. Therefore, the modification of these lifestyle-related problems could be strategies for coronary artery disease (CAD) and dementia prevention. While several pharmacological agents, including cholinesterase inhibitors [4], have been developed for dementia, sufficiently effective and curative treatments have not yet been established. Therefore, the identification of residual risk factors is important for dementia prevention.

The Japan Eicosapentaenoic Acid Lipid Intervention Study showed that long-term use of eicosapentaenoic acid (EPA) is effective for prevention of major coronary events in Japanese hypercholesterolemic patients [5]. In addition, recent studies demonstrated that consumption of fish and n-3 polyunsaturated fatty acids (PUFAs) reduced the incidence of cognitive impairment [6]. These studies indicate that a reduced serum level of $n-3$ PUFAs may be a risk factor for both CAD and cognitive impairment.

However, it is currently unknown whether reduced serum levels of n-3 PUFAs are associated with cognitive impairment, and more specifically, which components of PUFAs are associated with cognitive function in CAD patients. Therefore, the aim of this study was to investigate the association between cognitive function and n-3 PUFA levels (including eicosapentaenoic acid [EPA], docosahexaenoic acid [DHA], dihomogammalinolenic acid [DGLA], and arachidonic acid [AA]) in CAD patients, and to identify which components of PUFAs are associated with cognitive function in these patients.

We hypothesized that decreased level of EPA would be associated with cognitive impairment in patients with CAD.

\section{Material and methods Patients and study design}

In patients with CAD, serum PUFA levels were measured for identification of residual risk factors for CAD. In addition, patients underwent mini-mental state examinations (MMSE) to screen cognitive function [1]. We retrospectively reviewed 146 consecutive Japanese patients diagnosed with CAD in the Department of Cardiology at
Tokushima University Hospital between April 2013 and March 2014.

Patients with CAD were defined as patients with a history of myocardial infarction, angiographic evidence of at least $50 \%$ stenosis by area in at least 1 coronary artery, evidence of exercise-induced ischemia, or history of coronary revascularization. The exclusion criteria were as follows: use of fish oil supplements or n-3 fatty acidcontaining drugs or a history of myocardial infarction

\section{Table 1 Clinical characteristics of subjects}

\begin{tabular}{|c|c|}
\hline \multicolumn{2}{|l|}{ Variables } \\
\hline Number of patients & 146 \\
\hline Mini-mental state examination score & $27.8 \pm 3.1$ \\
\hline Male, n (\%) & $111(76.0 \%)$ \\
\hline Age, y & $70.9 \pm 8.6$ \\
\hline Body mass index, $\mathrm{kg} / \mathrm{m}^{2}$ & $23.5 \pm 3.4$ \\
\hline Systolic blood pressure, mmHg & $127 \pm 16.3$ \\
\hline Diastolic blood pressure, $\mathrm{mmHg}$ & $71 \pm 11.6$ \\
\hline Low-density lipoprotein cholesterol, mg/dL & $96.0 \pm 34.4$ \\
\hline Triglycerides, mg/dL & $124.5 \pm 70.3$ \\
\hline High-density lipoprotein cholesterol, mg/dL & $55.0 \pm 14.7$ \\
\hline $\mathrm{HbA1c} \%$ & $6.7 \pm 5.5$ \\
\hline Fasting plasma blood glucose, mg/dL & $130.0 \pm 58.7$ \\
\hline Brain natriuretic peptide, $\mathrm{pg} / \mathrm{mL}$ & $151.6 \pm 274.2$ \\
\hline Left ventricular ejection fraction, $\%$ & $58.6 \pm 11.2$ \\
\hline \multicolumn{2}{|l|}{ Fatty acids } \\
\hline Docosahexaenoic acid, $\mu \mathrm{g} / \mathrm{mL}$ & $132.5 \pm 52.2$ \\
\hline Eicosapentaenoic acid, $\mu \mathrm{g} / \mathrm{mL}$ & $68.9 \pm 40.4$ \\
\hline Arachidonic acid, $\mu \mathrm{g} / \mathrm{mL}$ & $173.0 \pm 43.4$ \\
\hline Dihomogammalinolenic acid, $\mu \mathrm{g} / \mathrm{mL}$ & $34.5 \pm 11.4$ \\
\hline \multicolumn{2}{|l|}{ Complications } \\
\hline Dyslipidemia, n (\%) & $126(86.3 \%)$ \\
\hline Hypertension, n (\%) & $131(89.7 \%)$ \\
\hline Diabetes mellitus, n (\%) & $80(54.8 \%)$ \\
\hline Cerebrovascular disease, n (\%) & $25(17.1 \%)$ \\
\hline Smoking, n (\%) & $108(74.0 \%)$ \\
\hline \multicolumn{2}{|l|}{ Drugs } \\
\hline ACEI/ARB, n (\%) & $91(62.3 \%)$ \\
\hline$\beta$-blockers, n (\%) & $53(36.3 \%)$ \\
\hline Calcium channel blockers, n (\%) & $62(42.5 \%)$ \\
\hline Statins, n (\%) & $123(84.2 \%)$ \\
\hline Ezetimibe, n (\%) & $8(5.5 \%)$ \\
\hline Diuretics loop, n (\%) & $27(18.5 \%)$ \\
\hline Mineralocorticoid antagonists, n (\%) & $7(4.8 \%)$ \\
\hline
\end{tabular}


within 1 month. In addition, patients with symptomatic active malignant diseases or liver dysfunction (aspartate aminotransferase levels >100 IU/L, alanine aminotransferase levels $>100$ IU/L) were also excluded.

Hypertensive patients were defined as those with a systolic blood pressure of $\geq 140 \mathrm{mmHg}$ and/or diastolic blood pressure of $\geq 90 \mathrm{mmHg}$ and/or individuals receiving antihypertensive medications. Dyslipidemic patients were defined as those with a low-density lipoprotein cholesterol level (LDL-C) of $\geq 140 \mathrm{mg} / \mathrm{dL}$, a triglyceride level of $\geq 150 \mathrm{mg} / \mathrm{dL}$, a high-density lipoprotein cholesterol level (HDL-C) of $<40 \mathrm{mg} / \mathrm{dL}$, or individuals receiving lipid-lowering medications. Diabetic patients were defined as individuals receiving insulin or oral hypoglycemic agents or those with an HbA1c level of $\geq 6.5 \%$, fasting plasma glucose level of $126 \mathrm{mg} / \mathrm{dL}$, or non-fasting plasma glucose level of $\geq 200 \mathrm{mg} / \mathrm{dL}$.

Serum fatty acid composition, including levels of EPA, DHA, DGLA, and AA, was measured by gas-liquid

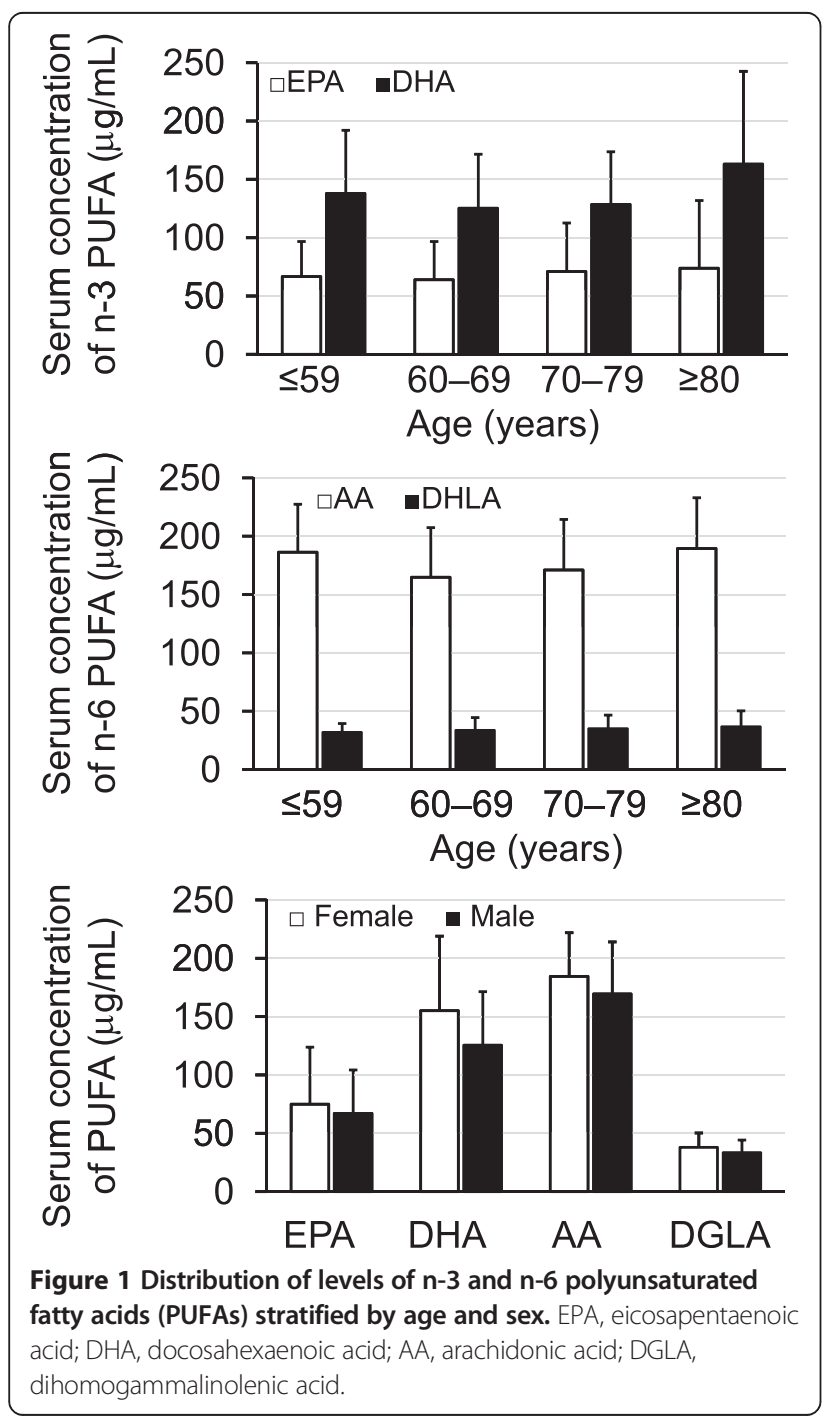

chromatography at a commercially available laboratory (SRL, Tokyo, Japan).

Since n-6 PUFAs, including AA, are often considered to be pro-inflammatory fatty acids and the EPA/AA ratio is associated with a low incidence of cardiac events [7], EPA/AA and DHA/AA ratios were also calculated.

In addition, other biochemical parameters, including LDL-C, HDL-C, triglycerides, fasting plasma glucose, and $\mathrm{HbA1c}$, were also measured.

Cardiac function was evaluated by measuring brain natriuretic peptide (BNP), a biomarker which represents left ventricular endodiastolic pressure, and left ventricular ejection fraction (LVEF) evaluated by echocardiography. The LVEF was calculated using the modified Simpson's method with the apical 4-chamber and 2-chamber views.

Cognitive function was evaluated by MMSE, which is widely used as a screening tool for assessment of cognitive function [8].

This study protocol was approved by the Tokushima University Hospital Ethics Committee.

\section{Statistical analysis}

Continuous variables were averaged and expressed as the mean \pm standard deviation, and categorical parameters were expressed as a percentage. MMSE scores and BNP levels were natural log transformed for statistical analysis because of non-normal distributions. Associations between CAD risk factors and MMSE scores were

Table 2 Pearson correlation analysis between MMSE scores and cardiovascular risk factors

\begin{tabular}{lll}
\hline Variables & R & P-value \\
\hline Age & -0.20 & $<0.01$ \\
Sex & - & 0.30 \\
Body mass index & 0.09 & 0.23 \\
DHA & 0.12 & 0.06 \\
EPA & 0.25 & $<0.01$ \\
AA & 0.1 & 0.99 \\
DGLA & -0.001 & 0.20 \\
EPA/AA & 0.22 & 0.01 \\
DHA/AA & 0.10 & 0.20 \\
Left ventricular ejection fraction & 0.15 & 0.04 \\
Brain natriuretic peptide & -0.28 & $<0.01$ \\
Hypertension & - & 0.96 \\
Dyslipidemia & - & 0.89 \\
Diabetes mellitus & - & 0.37 \\
Cerebrovascular disease & - & 0.79 \\
Current/previous smoker & - & 0.12 \\
\hline
\end{tabular}

MMSE, mini-mental state examination; DHA, docosahexaenoic acid; EPA, eicosapentaenoic acid; AA, arachidonic acid; DGLA, dihomogammalinolenic acid. 

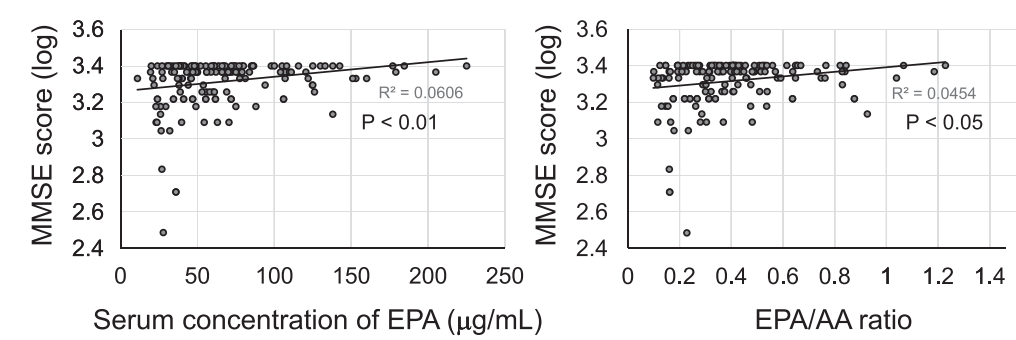

Figure 2 Association between serum concentration of eicosapentaenoic acid (EPA) and EPA/arachidonic acid (AA) ratio and mini-mental state examination (MMSE) score.

determined by the Student's t-test or the Pearson's correlation analysis. Multiple regression analysis (standard least squares method) was used to assess the degrees of association between the CAD risk factor variables and the MMSE scores. Residuals and residual vs. fit plots were examined to ensure homoscedasticity.

All statistical analyses were performed using JMP 10 software. Statistical significance was defined as $\mathrm{P}<0.05$.

\section{Results}

\section{Clinical characteristics of subjects}

Patient characteristics are shown in Table 1. The mean MMSE score was $27.8 \pm 3.1$, indicating that subjects generally had mild cognitive impairment. The serum concentration of n-3 PUFAs, including EPA and DHA, and n-6 PUFAs, including AA and DGLA, are shown in Figure 1 by patient age and sex. EPA and DHA concentrations were the highest among patients aged $\geq 80$ years, and levels of EPA, DHA, DGLA, and AA were higher in women than in men.

\section{Correlation between n-3 PUFAs and MMSE score}

The Pearson correlation analysis showed that levels of EPA, EPA/AA ratio, and LVEF were positively associated with MMSE score, and that age and BNP was inversely associated with MMSE score (Table 2, Figure 2). There were no relationships between MMSE score and body mass index or levels of DHA, AA, DGLA, and DHA/AA ratio (Figure 3 ). In addition, there were no differences in MMSE scores according to patient sex and risk factors, including hypertension, dyslipidemia, diabetes mellitus, cerebrovascular disease, and history of current/previous smoking. Interestingly, both LVEF and BNP (parameters of cardiac function) were associated with serum levels of DHA and DHA/AA but not EPA or EPA/AA (Additional file 1: Tables S1 and S2).

Multiple regression analysis was then performed to elucidate independent determinants of MMSE score; age ( $\mathrm{P}<0.05)$ was negatively associated with MMSE score, while EPA levels $(\mathrm{P}<0.01)$ and EPA/AA ratio $(\mathrm{P}<0.05)$ were positively associated with MMSE score. However, sex; body mass index; levels of DHA, AA, DGLA, and DHA/AA ratio; LVEF; levels of BNP; presence of hypertension, dyslipidemia, diabetes mellitus, and cerebrovascular disease; and history of current/previous smoking were statistically excluded (Table 3 ).

\section{Discussion}

The present study demonstrated that decreased serum levels of EPA and a reduced EPA/AA ratio are associated with cognitive impairment in patients with CAD, indicating that decreased EPA is a risk factor for development of cognitive impairment this patient population. AA levels showed no association with MMSE scores, while low EPA levels were independently correlated with MMSE scores. Therefore, EPA/AA did not increase the predictive power of EPA alone. In addition, the Pearson correlation analysis exhibited an association between MMSE scores and LVEF or BNP; however, multiple regression in an age-adjusted model showed no association.
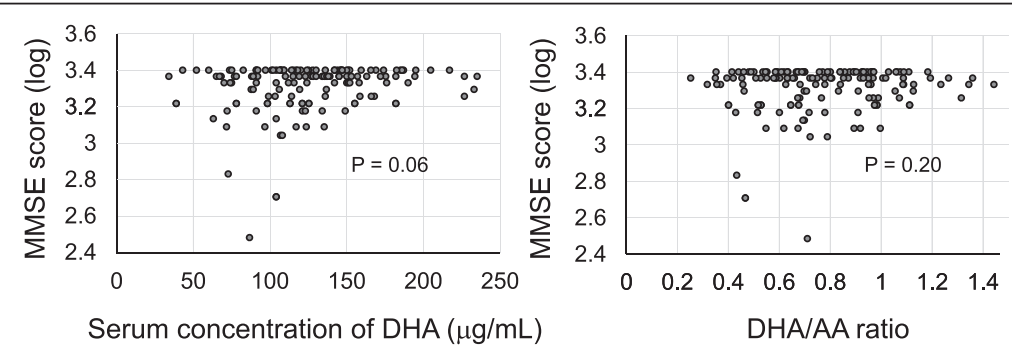

Figure 3 There were no associations between serum concentration of docosahexaenoic acid (DHA) and DHA/AA ratio and mini-mental state examination (MMSE) score. 
Table 3 Multiple regression analysis for determinants of degree of MMSE score in patients with CAD

\begin{tabular}{|c|c|c|c|}
\hline Variables & Coefficient & $\begin{array}{l}95 \% \text { confidence } \\
\text { interval }\end{array}$ & P-value \\
\hline \multicolumn{4}{|l|}{ Model 1} \\
\hline Age, years & -0.004 & -0.007 to -0.001 & 0.02 \\
\hline Male sex & 0.005 & -0.03 to 0.04 & 0.76 \\
\hline Body mass index, $\mathrm{kg} / \mathrm{m}^{2}$ & -0.0008 & -0.001 to 0.007 & 0.85 \\
\hline Hypertension & 0.02 & -0.01 to 0.06 & 0.24 \\
\hline Dyslipidemia & -0.001 & -0.04 to 0.03 & 0.91 \\
\hline Diabetes mellitus & 0.001 & -0.02 to 0.02 & 0.98 \\
\hline Cerebrovascular disease & -0.01 & -0.04 to 0.02 & 0.50 \\
\hline Current/previous smoker & 0.001 & -0.03 to 0.04 & 0.80 \\
\hline $\mathrm{EPA}, \mu \mathrm{g} / \mathrm{mL}$ & 0.001 & 0.0003 to 0.002 & $<0.01$ \\
\hline $\mathrm{DHA}, \mu \mathrm{g} / \mathrm{mL}$ & -0.0004 & -0.001 to 0.0003 & 0.22 \\
\hline $\mathrm{AA}, \mu \mathrm{g} / \mathrm{mL}$ & 0.0001 & -0.0001 to 0.001 & 0.83 \\
\hline $\mathrm{DGLA}, \mu \mathrm{g} / \mathrm{mL}$ & 0.001 & -0.002 to 0.004 & 0.45 \\
\hline LVEF, \% & 0.001 & -0.001 to 0.004 & 0.20 \\
\hline $\mathrm{BNP}, \mathrm{pg} / \mathrm{mL}$ & -0.015 & -0.035 to 0.005 & 0.15 \\
\hline \multicolumn{4}{|l|}{ Model 2} \\
\hline Age, years & -0.003 & -0.006 to -0.0002 & 0.04 \\
\hline Male sex & 0.002 & -0.03 to 0.04 & 0.89 \\
\hline Body mass index, $\mathrm{kg} / \mathrm{m}^{2}$ & 0.0008 & -0.006 to 0.008 & 0.83 \\
\hline Hypertension & 0.02 & -0.02 to 0.05 & 0.37 \\
\hline Dyslipidemia & 0.003 & -0.03 to 0.04 & 0.84 \\
\hline Diabetes mellitus & 0.002 & -0.03 to 0.04 & 0.85 \\
\hline Cerebrovascular disease & -0.01 & -0.03 to 0.02 & 0.58 \\
\hline Current/previous smoker & 0.003 & -0.03 to 0.04 & 0.85 \\
\hline EPA/AA & 0.15 & 0.03 to 0.28 & 0.01 \\
\hline DHA/AA & -0.04 & -0.15 to 0.07 & 0.42 \\
\hline LVEF, \% & 0.001 & -0.001 to 0.003 & 0.29 \\
\hline $\mathrm{BNP}, \mathrm{pg} / \mathrm{mL}$ & -0.018 & -0.04 to 0.001 & 0.06 \\
\hline
\end{tabular}

Model $1 \mathrm{R}^{2}=0.20 ; \mathrm{P}<0.05$

Model $2 \mathrm{R}^{2}=0.17 ; \mathrm{P}<0.05$.

EPA, eicosapentaenoic acid; DHA, docosahexaenoic acid; $A A$, arachidonic acid; DGLA, dihomogammalinolenic acid; LVEF, left ventricular ejection fraction; $\mathrm{BNP}$, brain natriuretic peptide.

Although there is a possibility of confounding between cardiac function and age, our results indicate that MMSE scores were associated with EPA levels independent of cardiac function.

It has been reported that fish intake is associated with cognitive function $[9,10]$, and n-3 PUFA-containing food improves cognitive function [11]. The exact mechanisms of EPA on neuronal function are unknown; however, EPA is involved in endothelial-dependent vascular function and plays important roles in the prevention of microcirculatory insufficiency. Therefore, one possible mechanism is that decreased EPA results in microcirculation insufficiency in the brain, thus leading to ischemia-induced brain damage. It has been reported that EPA enhances endothelial expression of nitric oxide synthase, and activation of endothelial nitric oxide synthase leads to vasodilation and endothelial protection [12,13]. Reduced nitric oxide synthase-induced microcirculation insufficiency may be involved in the development of cognitive impairment. Brain microvascular insufficiency, including cerebral white matter disease, is an early marker of cognitive impairment $[14,15]$. Thus, in patients with very mild cognitive impairment, such as those who were included in this study, microcirculation insufficiency rather than amyloid $\beta$ deposition, the cause of Alzheimer's disease, may be the predominant contributor to vascular dementia.

It has been reported that vascular inflammation is associated with cognitive function [16]. EPA is known to have the ability to attenuate tumor necrosis factor- $\alpha$-induced upregulation of vascular cell adhesion molecule-1, intercellular adhesion molecule-1, and monocyte chemoattractant protein-1 [17]. The anti-inflammatory effects of EPA might contribute to the prevention of cognitive impairment.

A previous meta-analysis demonstrated that subjects with predementia syndrome had significantly lower levels of EPA but not DHA or total n-3 PUFA [18]; thus, serum EPA may be a more sensitive biomarker for prediction of cognitive impairment compared to serum DHA. It has also been reported that administration of n-3 PUFA to patients with very mild Alzheimer's disease (MMSE >27 points) delayed the rate of cognitive dysfunction, indicating the importance of n-3 PUFA on brain function in early stages of cognitive impairment [19].

In addition, EPA is converted into DHA in the liver and at the blood-brain barrier and has been shown to cross the blood-brain barrier after dietary supplementation, thereby suggesting that EPA plays important roles in cognitive function after conversion into DHA [20,21]. EPA could be a rich source of DHA and may compensate for reduced levels of DHA in the brain; decreased serum EPA may thus be a more profound indicator of cognitive function than decreased serum DHA.

The present study had several limitations. In particular, this was a retrospective study with a small sample size in a single center. Larger clinical cohort studies are needed to clarify the effects of EPA on cognitive function.

\section{Conclusions}

Serum EPA concentration rather than serum DHA concentration is associated with cognitive function in patients with CAD, suggesting that a low serum EPA level is a risk factor for cognitive impairment independent of cardiac function. Our data support a role of dietary n-3 PUFAs in preventing the cognitive decline in CAD patients. 


\section{Additional file}

Additional file 1: Table S1. Pearson correlation analysis between left ventricular ejection fraction and serum levels of polyunsaturated fatty acids. Table S2. Pearson correlation analysis between brain natriuretic peptide levels and serum levels of polyunsaturated fatty acids.

\section{Abbreviations}

PUFAs: Polyunsaturated fatty acids; EPA: Eicosapentaenoic acid; DHA: Docosahexaenoic acid; DGLA: Dihomogammalinolenic acid; AA: Arachidonic acid; CAD: Coronary artery disease; MMSE: Mini-mental state examinations; LDL-C: Low-density lipoprotein cholesterol level; HDL-C: Highdensity lipoprotein cholesterol level.

\section{Competing interests}

The authors declare that they have no conflicts of interest to disclose.

\section{Authors' contributions}

SY, TH, RU, DF, AT, JH, T Ise, KY, TT, T Iwase, HY, TS, TW, M Shimabukuro, MA M Sata collected data and SY analyzed the data and wrote the manuscript. KA, MA, M Shimabukuro, and M Sata provided the suggestion for this study. All authors read and approved the final manuscript.

\section{Acknowledgements}

We thank the staff of the Hospital Information Center of Tokushima University Hospital for abstracting patients' data from medical records. This work was supported in part by JSPS KAKENHI (Grant Number 25461131 and 25860605) and Actelion Academia Prize 2013.

\section{Author details}

${ }^{1}$ Department of Cardiovascular Medicine, The University of Tokushima Graduate School of Health Biosciences, 3-18-15 Kuramoto-cho, Tokushima-city, Tokushima 770-8503, Japan. ²Department of Medicine and Bioregulatory Sciences, The University of Tokushima Graduate School of Health Biosciences, 3-18-15 Kuramoto-cho, Tokushima-city, Tokushima 770-8503, Japan. ${ }^{3}$ Department of Cardio-Diabetes Medicine, The University of Tokushima Graduate School of Health Biosciences, 3-18-15 Kuramoto-cho, Tokushima-city, Tokushima 770-8503, Japan. ${ }^{4}$ Department of Medical Education, The University of Tokushima Graduate School of Health Biosciences, 3-18-15 Kuramoto-cho, Tokushima-city, Tokushima 770-8503, Japan.

Received: 22 July 2014 Accepted: 6 November 2014

Published: 4 December 2014

\section{References}

1. Kovacic JC, Castellano JM, Fuster V: The links between complex coronary disease, cerebrovascular disease, and degenerative brain disease. Ann N Y Acad Sci 2012, 1254:99-105.

2. Schmidt SL, Correa PL, Tolentino JC, Manhaes AC, Felix RM, Azevedo JC, Barbirato GB, Mendes MHF, Boechat Y, Cabral H, Schmidt GJ, Dohmann HF, Mesquita CT: Value of combining activated brain FDG-PET and cardiac MIBG for the differential diagnosis of dementia - Differentiation of dementia with Lewy bodies and Alzheimer disease when the diagnoses based on clinical and neuroimaging criteria are difficult. Clin Nucl Med 2008, 33:398-401.

3. O'Donnell M, Teo K, Gao P, Anderson C, Sleight P, Dans A, Marzona I, Bosch J, Probstfield J, Yusuf S: Cognitive impairment and risk of cardiovascular events and mortality. Eur Heart J 2012, 33:1777-1786.

4. Anand $P$, Singh B: A review on cholinesterase inhibitors for Alzheimer's disease. Arch Pharm Res 2013, 36:375-399.

5. Yokoyama M, Origasa H, Matsuzaki M, Matsuzawa $Y$, Saito $Y$, Ishikawa $Y$, Oikawa S, Sasaki J, Hishida H, Itakura H, Kita T, Kitabatake A, Nakaya N, Sakata T, Shimada K, Shirato K: Japan, E. P. A. lipid intervention study Investigators: Effects of eicosapentaenoic acid on major coronary events in hypercholesterolaemic patients (JELIS): a randomised open-label, blinded endpoint analysis. Lancet 2007, 369:1090-1098.

6. Morris MC, Evans DA, Bienias JL, Tangney CC, Bennett DA, Wilson RS, Aggarwal N, Schneider J: Consumption of fish and n-3 fatty acids and risk of incident Alzheimer disease. Arch Neurol 2003, 60:940-946.
7. Matsuzaki M, Yokoyama M, Saito Y, Origasa H, Ishikawa Y, Oikawa S, Sasaki J, Hishida H, Itakura H, Kita T, Kitabatake A, Nakaya N, Sakata T, Shimada K, Shirato K, Matsuzawa Y, Japan Jl: Incremental Effects of Eicosapentaenoic Acid on Cardiovascular Events in Statin-Treated Patients With Coronary Artery Disease - Secondary Prevention Analysis From JELIS. Circ J 2009, 73:1283-1290.

8. Folstein MF, Folstein SE, McHugh PR: "Mini-mental state". A practical method for grading the cognitive state of patients for the clinician. J Psychiatr Res 1975, 12:189-198.

9. Albanese E, Dangour AD, Uauy R, Acosta D, Guerra M, Guerra SSG, Huang YQ, Jacob KS, de Rodriguez JL, Noriega LH, Salas A, Sosa AL, Sousa RM, Williams J, Ferri CP, Prince MJ: Dietary fish and meat intake and dementia in Latin America, China, and India: a 10/66 Dementia Research Group population-based study. Am J Clin Nutr 2009, 90:392-400.

10. Gillette-Guyonnet S, Van Kan GA, Andrieu S, Barberger-Gateau P, Berr C, Bonnefoy M, Dartigues JF, De Groot L, Ferry M, Galan P, Hercberg S, Jeandel C, Morris MC, Nourhashemi F, Payette H, Poulain JP, Portet F, Roussel AM, Ritz P, Rolland Y, Vellas B: lana task force on nutrition and cognitive decline with aging. J Nutr Health Aging 2007, 11:132-152.

11. Scheltens P, Kamphuis PJGH, Verhey FRJ, Rikker MGMO, Wurtman RJ, Wilkinson D, Twisk JWR, Kurz A: Efficacy of a medical food in mild Alzheimer's disease: A randomized, controlled trial. Alzheimers Dementia 2010, 6:1-10.

12. Tardivel S, Gousset-Dupont A, Robert V, Pourci ML, Grynberg A, Lacour B Protective Effects of EPA and Deleterious Effects of DHA on eNOS Activity in Ea hy 926 Cultured with Lysophosphatidylcholine. Lipids 2009, 44:225-235.

13. Chen $\mathrm{JH}$, Shearer $\mathrm{GC}$, Chen $\mathrm{QH}$, Healy $\mathrm{CL}$, Beyer AJ, Nareddy VB, Gerdes AM, Harris WS, O'Connell TD, Wang DJ: Omega-3 Fatty Acids Prevent Pressure Overload-Induced Cardiac Fibrosis Through Activation of Cyclic GMP/Protein Kinase G Signaling in Cardiac Fibroblasts. Circulation 2011, 123:584-593.

14. Mortamais M, Artero S, Ritchie K: Cerebral white matter hyperintensities in the prediction of cognitive decline and incident dementia. Int Rev Psychiatry 2013, 25:686-698.

15. Kandiah N, Chander R, Zhang A, Yee CC: Cerebral white matter disease is independently associated with BPSD in Alzheimer's disease. J Neurol Sci 2014, 337:162-166.

16. Yagi $S$, Akaike $M$, Ise $T$, Ueda $Y$, Iwase $T$, Sata M: Renin-angiotensinaldosterone system has a pivotal role in cognitive impairment. Hypertens Res 2013, 36:753-758.

17. Matsumoto M, Sata M, Fukuda D, Tanaka K, Soma M, Hirata Y, Nagai R: Orally administered eicosapentaenoic acid reduces and stabilizes atherosclerotic lesions in ApoE-deficient mice. Atherosclerosis 2008, 197:524-533.

18. Lin PY, Huang SY, Su KP: A meta-analytic review of polyunsaturated fatty acid compositions in patients with depression. Biol Psychiatry 2010, 68:140-147.

19. Freund-Levi $Y$, Eriksdotter-Jonhagen M, Cederholm T, Basun H, Faxen-Irving G, Garlind A, Vedin I, Vessby B, Wahlund LO, Palmblad J: Omega-3 fatty acid treatment in 174 patients with mild to moderate Alzheimer disease: OmegAD study: a randomized double-blind trial. Arch Neurol 2006, 63:1402-1408.

20. Freund Levi Y, Vedin I, Cederholm T, Basun H, Faxen Irving G, Eriksdotter M, Hjorth E, Schultzberg M, Vessby B, Wahlund LO, Salem N Jr, Palmblad J: Transfer of omega-3 fatty acids across the blood-brain barrier after dietary supplementation with a docosahexaenoic acid-rich omega-3 fatty acid preparation in patients with Alzheimer's disease: the OmegAD study. J Intern Med 2014, 275:428-436.

21. Moore SA: Polyunsaturated fatty acid synthesis and release by brain-derived cells in vitro. J Mol Neurosci 2001, 16:195-200.

\section{doi:10.1186/1475-2891-13-112}

Cite this article as: Yagi et al:: Serum concentration of eicosapentaenoic acid is associated with cognitive function in patients with coronary artery disease. Nutrition Journal 2014 13:112. 